Fi nite-si ze dependence of the bri dge functi on ext racted from nol ecul ar dynamics si mul at i ons

\begin{tabular}{|l|l|}
\hline 著者 & Baunket ner A., H wat ar i Yasuaki \\
\hline $\begin{array}{l}\text { j our nal or } \\
\text { publ i cat i on t i t l e }\end{array}$ & Physi cal Revi ew E \\
\hline vol une & 63 \\
\hline number & 6 \\
\hline page $r$ ange & $061201-1-061201-4$ \\
\hline year & 2001- 06-01 \\
\hline URL & ht t p: //hdl . handl e. net /2297/1706 \\
\hline
\end{tabular}




\title{
Finite-size dependence of the bridge function extracted from molecular dynamics simulations
}

\author{
A. Baumketner* and Y. Hiwatari \\ Faculty of Science, Kanazawa University, Kakuma, Kanazawa, 920-1192, Japan
}

(Received 30 November 2000; published 11 May 2001)

\begin{abstract}
The bridge function for liquid sodium at $T=373 \mathrm{~K}$ is obtained by using the mean spherical approximation to extrapolate the pair distribution function (PDF), calculated in molecular dynamics (MD) simulations, beyond the half simulation box length for two sizes of the MD system. The bridge function is found to strongly depend on the total number of particles used in the simulation cell. This dependency leads to a spurious maximum of the static structure factor at long wavelengths, obtained from the reference hypernetted-chain approximation (RHNC) with the MD system used as a reference system (RHNC-MD). A simple self-consistent procedure, proposed to account for the finite-size effects in the bridge function, allows one to efficiently correct the RHNC-MD static structure factor for all unphysical manifestations.
\end{abstract}

DOI: 10.1103/PhysRevE.63.061201

PACS number(s): 61.20.Ja, 61.25.Mv

\section{INTRODUCTION}

In recent years, the reference hypernetted-chain approximation (RHNC) has proven to be one of the most successful approaches in the theory of liquid state [1]. The key input quantity in this approximation is the system's bridge function $B(r)$. Bridge functions constructed from the ansatz of the hard sphere (HS) system- so-called variational modified hypernetted-chain approximation (VMHNC) - were found to produce very reasonable agreement with the molecular dynamics (MD) results for the pair distribution functions (PDF) in a wide class of liquids. For some specific systems, however, the success of the VMHNC method is not quite as spectacular [2-4]. The main cause of the failure of the VMHNC in those systems is assigned to the principal incapability of the HS-based bridge function to mimic the intermediate-distance part of actual $B(r)$, which depends on the details of the underlying interparticle potential. As an alternative approach to the VMHNC for such "troubled" systems, a procedure of extracting bridge function from molecular dynamics simulations was proposed (see Ref. [2], and references therein). The extraction procedure consists in solving the Ornstein-Zernike (OZ) integral equation [1]

$$
h(r)=c(r)+\rho \int c\left(\left|\vec{r}-\vec{r}^{\prime}\right|\right) h\left(r^{\prime}\right) d \vec{r}^{\prime},
$$

supplemented by the following closure:

$$
\begin{gathered}
g(r)=g^{N}(r), \quad r<R, \\
c(r)=-\beta U_{c}(r), \quad r>R,
\end{gathered}
$$

where $c(r)$ is the direct correlation function $\gamma(r)=h(r)$ $-c(r), h(r)$ is the pair correlation function, $g(r)=h(r)$ +1 is the pair distribution function. In Eq. (2), $\beta$ denotes the inverse temperature $1 / k_{B} T$ with the Boltzmann constant $k_{B}$ and the temperature $T, U_{c}(r)$ is the cutoff two-particle inter-

\footnotetext{
*Permanent address: Institute for Condensed Matter Physics, 1 Svientsitsky St., Lviv 79011, Ukraine.
}

atomic potential used in the simulation, and $\rho$ is the particle density of the system. The superscript $N$ over $g(r)$ in Eq. (2) indicates that this function is obtained from a molecular dynamics ensemble of $N$ particles. The capital $R$ in the righthand side of Eq. (2) stands for the extrapolation distance of the PDF which is limited to half the simulation box length $L / 2$.

Molecular dynamics system interacting via the cutoff potential $U_{c}(r)$ can be considered as a reference system within the RHNC approach. The bridge function $B(r)$ of such a reference system is calculated from the solution of Eqs. (1) and (2) as follows:

$$
B(r)=\gamma(r)-\ln g(r)-\beta U_{c}(r)
$$

We note that relation (3) is valid for both short distances $r$ $<R$ and long distances $r>R$. It was shown earlier that in liquid metals, the bridge function depends only weakly on the details of the long-range part of the interparticle potential $[3,4]$. This means that the bridge function calculated for a model MD system can be used to recover the structure of the full system, interacting via uncut potential $U(r)$, by solving the $\mathrm{OZ}$ equation complemented by the closure

$$
g(r)=\exp [\gamma(r)-\beta U(r)+B(r)] .
$$

We will refer to this method of calculating the PDF as the RHNC-MD approach. It efficiently corrects $g^{N}(r)$ for the inevitable error caused by cutting off the interparticle potential at a finite distance in simulations, as was shown in Ref. [4] for the case of liquid aluminum. The RHNC-MD method is an especially powerful device for liquid structure calculations when applied to small MD systems, for which computational cost of simulations is not high. This fact motivated the authors of Refs. [2-4] to study the bridge function dependence on the simulation box size. The aim of this paper is to investigate the impact of a phenomenon disregarded previously $[3,4]$ - finite-size effects in computer experiments [5] — on computer-generated bridge functions. Since the finite-size effects become increasingly relevant for small MD systems and their influence on $B(r)$ or the static structure is not known a priori, the subject of the present study is of 
great current interest. In the following, we give a short outline of a self-consistent procedure devised to correct $B(r)$ for finite-size effects.

It is well known that a pair distribution function $g^{N}(r)$ obtained in a closed ensemble with fixed number of particles $N$, such as, for example, the molecular dynamics ensemble, differs from its open system, i.e., where $N$ is allowed to fluctuate, counterpart $g(r)$. The two functions coincide in the thermodynamic limit $N \rightarrow \infty$ only and the difference between them at finite $N$ is called finite-size effects in computer simulations. Clearly, if extracted according to Eqs. (1)-(3) the bridge function $B(r)$ will depend on $N$ through implicit dependence on $g^{N}(r)$. Two other quantities on which $B(r)$ depends are the potential energy truncation distance $R_{c}$ and the PDF extrapolation distance $R$, both being thoroughly studied in Refs. [3,4]. We note that, in principle, closure (2) is not correct since it contains a closed system PDF at $r$ $<R$ and the open system PDF (in the mean spherical approximation) at $r>R$. This improperness can be corrected for by transforming obtained in a simulation $g^{N}(r)$ into the open system PDF, $g(r)$, i.e., correcting $g^{N}(r)$ for finite-size effects, before substituting it into Eq. (2). The simplest possible expression relating the PDFs in open and closed systems can be written as [5]

$$
g(r)=g^{N}(r)+\frac{1}{N} \frac{S(0)}{2} \frac{\partial^{2}}{\partial \rho^{2}}\left[\rho^{2} g^{N}(r)\right]
$$

where $S(0)$ is the static structure factor of the open system at $k=0$. Relation (5) has the standard for transforming between ensembles correction term of order $1 / N$. Its explicit evaluation from the MD method, however, is not practical or, in some cases, it is even infeasible. Thus we choose a further simplification of Eq. (5) by neglecting the density dependence of $g(r)$ :

$$
g(r)=g^{N}(r)+\frac{S(0)}{N} g^{N}(r) .
$$

Now this new expression (6) is well suitable for practical applications. Provided $S(0)$ is known, Eq. (6) could be used for correcting the PDF obtained from an MD simulation. $S(0)$ is not known a priori, however, but linked to $g(r)$ itself by standard Fourier transformation [1]. The fact that $S(k)$ and $g(r)$ are related makes it possible to construct an iterative cycle for evaluating $S(0)$. It consists of alternate application of the correction to PDF due to Eq. (6) for $r$ $<R$ and subsequent evaluation of this PDF for the whole range of distances due to Eqs. (1) and (2). Initially we adopt some trial guess for $S(0)$, which we obtained from the MoriHoshino-Watabe scheme [6], and correct $g^{N}(r)$ according to Eq. (6). Next, the corrected $g^{N}(r)$ is substituted into closure (2) and the OZ equation, supplemented by this closure, is solved numerically. The resulting solution $g(r)$ is Fourier transformed to calculate new estimate for $S(0)$ and the whole cycle is iterated until a desired convergence is reached. As a result we obtain $B(r), g(r)$, and $S(k)$ of the model system interacting via cutoff potential. In order to evaluate the structure functions of the full system one needs to use the obtained $B(r)$ in the RHNC scheme. We will refer to the just described method as iterative RHNC-MD method to distinguish it from the noniterative one given above in the text. The method was applied earlier by one of the authors to study the structure of a liquid potassium-sodium alloy [7]. In this report we test its efficiency for the case of a model monatomic liquid, liquid sodium near freezing.

\section{RESULTS AND DISCUSSION}

In order to assess the finite-size dependence of the bridge functions extracted from molecular dynamics simulations according to the procedure described above we performed two MD runs for model liquid sodium at a temperature $T$ $=373 \mathrm{~K}$ and density $\rho=0.024217 \AA^{-3}$ in standard $N V E$ ensembles of $N=500$ and 2048 particles. The pair potential was obtained from the second order perturbation theory in the empty-core local pseudopotential with the only parameter $r_{c}$ set to 1.78 a.u. [8]. The local field correction factor was used in the form of Ichimaru and Utsumi [9]. The potential energy was truncated at its third maximum $r=13.14 \AA$ so as to lift the force discontinuity at the truncation distance, and then shifted at that point to zero. In this way we made both force and potential energy fields continuous at the cutoff distance hence improving total energy conservation during the simulations. To eliminate the potential cutoff part from the overall dependency of the bridge function on $N$, we used the same potential truncation distance for both simulations. The equations of motion were integrated by the velocityVerlet algorithm with the time increment $\delta t=6$ fs. For the first MD system we performed $10^{6}$ time steps after initial equilibration and the second system was let to evolute over a period of $5 \times 10^{5}$ time steps. The selected lengths of the simulations allowed us to achieve the total number of PDF samples, which is proportional to $N(N-1)$ and the number of sampled configurations, to be of order $10^{10}-10^{11}$ as indicated in Ref. [4] necessary for canceling statistical noise in the PDF. In our simulations we sampled PDF every 10 time steps. For the PDF extrapolation distances $R$ from Eq. (2) we used the largest values possible, i.e., about $L / 2$, in both simulations.

Pair distribution functions obtained from the simulations were used to calculate bridge functions $B(r)$ according to the iterative procedure described above. To solve the $\mathrm{OZ}$ integral equation we used a Newton-Raphson routine [10] based on numerically inexpensive fast Fourier transforms. The number of grid points of the potential and step size in numerical integrations were 1024 points and $0.06 \AA$, respectively. The truncation distance used in the integrations $61.44 \AA$ is large enough to neglect the influence of the cutoff part of the potential on the long-wavelength limit of $S(k)$ (at least in the $\mathrm{HNC}$ and mean spherical approximations). The iterations in the Eqs. (1),(2),(6) cycle were stopped after two successive values of $S(0)$ did not differ more than by $0.01 \%$ of their magnitude. A total of 10 iterations were needed to achieve the desired self-consistency for both MD system sizes. In Fig. 1 we show the converged $B^{c}(r)$ and raw (without finitesize corrections) $B^{r}(r)$ bridge functions for the two systems 


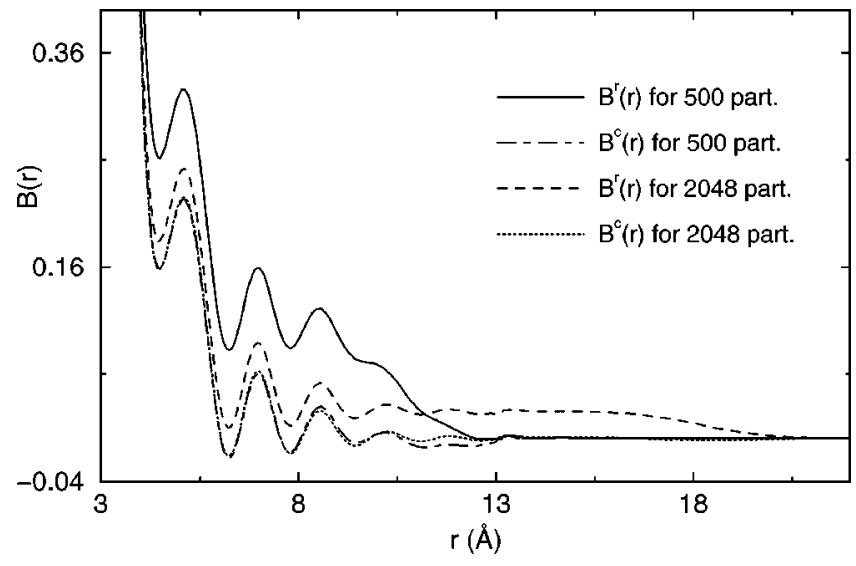

FIG. 1. Converged and raw bridge functions of liquid sodium at $T=373 \mathrm{~K}$ as extracted from molecular dynamics simulations of 500- and 2048-particle ensembles.

considered. The converged functions almost coincide with each other throughout the whole range of distances but for a small exception at $r \sim 12 \AA$, where the smaller system's $B(r)$ takes insignificant negative values. The raw bridge functions of both systems experience appreciable deviation from $B^{c}(r)$ for all $r$. In the region of small separations the deviation becomes negligible thus providing support for the universality hypothesis of Rosenfeld [11]. At intermediate distances, $B^{r}(r)$ 's of both MD systems are shifted upwards: the bigger the system the smaller the shift. The raw bridge function of the 2048-particle system additionally has a plateau extending from $r \sim 12 \AA$ up to the extrapolation distance $R \sim 20 \AA$. For larger separations $r>R, B^{r}(r)$ smoothly drops to zero. This behavior of the bridge function agrees well with the reported earlier results for liquid aluminum and LJ system [4]. As the extrapolation distance of the smaller system is only $\sim 13 \AA$ its raw bridge function does not exhibit any plateau. The authors of Ref. [4] conjectured that the appearance of the plateau in $B^{r}(r)$ is connected purely with insufficient statistics of the MD PDF and the noise that this function contains. They also found that by improving the PDF statistics, either by increasing the number of particles in the simulation cell or the length of the simulation, one can, in principle, reduce the plateau to zero. It was established that a total number of $10^{10}-10^{11}$ PDF samples was sufficiently large to totally cancel random statistical noise in PDF and remove the plateau. In our study we achieved comparable numbers of the PDF samples but the plateau did not disappear. Upon decreasing the total number samples the bridge function became more noisy but the plateau did not vanish. We are inclined to conclude, therefore, that the mentioned plateau is not connected with the random statistical error, associated with the number of samples, but with a sort of systematic error present in computer-generated PDF, i.e., finite-size effects. Correcting for the finite-size effects efficiently diminishes the plateau for all values of the extrapolation distance $R$ (in Fig. 1 only the data for $R \sim L / 2$ are shown).

Now we move on to discussing the finite-size effects on the structural properties - pair distribution function and static structure factor of the investigated system. For this

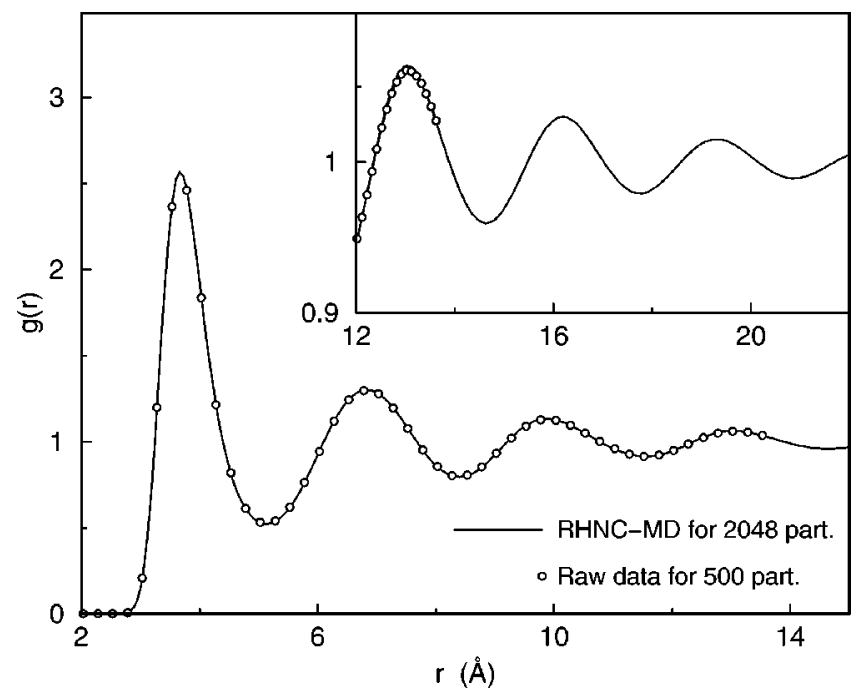

FIG. 2. The RHNC-MD pair distribution function for the 2048particle system and raw MD data for the 500-particle system. In the inset we present the long-range region of $g(r)$ in more detail.

purpose it is instructive to estimate the magnitude of the corrections to PDF due to relation (6). By substituting a typical for alkali metals value 0.05 for $S(0)$ and adopting typical number of particles in simulation box to be 500, we find the correction to be about $10^{-4}$ at long distances, where $g(r)$ $\sim 1$. Clearly, this quantity is too small to be visually observed on graphs of the PDF's obtained in the two simulations. In Fig. 2 we plotted the corrected PDF's computed from the iterative RHNC-MD scheme when using the 2048particle MD ensemble as a reference system and the raw MD data calculated for the 500-particle system. It is seen from the figure that the raw and corrected PDF are not distinguishable from each other for all separations. Much bigger sensitiveness to the details of the bridge function is displayed by

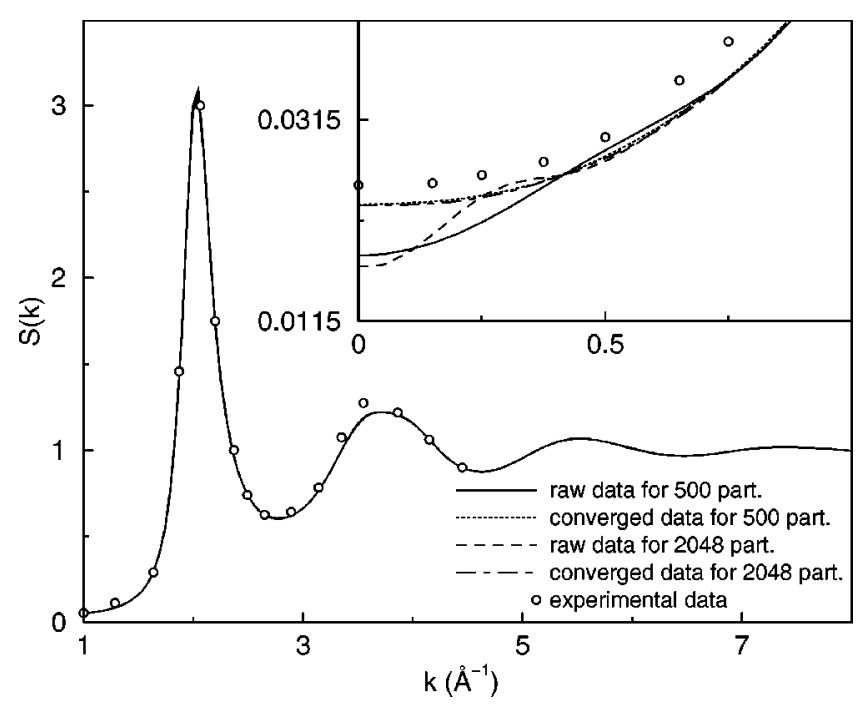

FIG. 3. The RHNC-MD static structure factors as obtained with the help of 500-and 2048-particle systems and the raw $S(k)$ for both systems. By open circles we denoted experimental data of Waseda [12]. 
the static structure factor. As seen from Fig. 3, in which we show the corrected (via iterative RHNC-MD procedure) and the raw static structure factors (via noniterative RHNC-MD method) obtained for the two MD systems, the corrected data perfectly coincide with each other. It is important to note here that while corrected $S(k)$ is the static structure factor of the open system interacting via full potential, the raw $S(k)$ has no physical meaning. In particular, it should not be confused with the static structure factor of the closed MD system. The largest departure of the raw static structure factors occurs in the region of small wave numbers $k \sim 0.5 \AA^{-1}$. In that region both raw $S(k)$ 's exhibit additional unphysical maximum. This maximum results from the intermediatedistance deviations of the bridge function and is totally removed upon applying the correction procedure. We estimate the order of the error brought about by neglecting the finitesize effects in the noniterative RHNC-MD method to be about $35 \%$ at $k=0$ for the system studied. For a comparison, in Fig. 3 we also depicted the experimental data of Waseda [12] for the static structure factor of liquid sodium under similar thermodynamic conditions as those of the present study. We see that the theoretical data agrees reasonably well with the actual static structure at all values of wave number.
Both the region of the main peak of $S(k)$ and the longwavelength region are accurately reproduced.

As conclusions we would like to make the following observations. The procedure of extracting bridge functions from molecular dynamics simulations proposed earlier [2] and, based upon it, the noniterative RHNC-MD method are badly affected by finite-size effects in computer experiments. The corrections to MD-generated PDF's caused by these effects are not visible on the pair distribution functions but have profound impact on the bridge functions and the static structure factor. In the long-wavelength limit they lead to a spurious maximum in $S(k)$. A simple self-consistent routine proposed in the paper provides a reasonable account of the finite-size effects and makes it possible to avoid unphysical manifestations in MD simulations of the liquid static structure.

\section{ACKNOWLEDGMENTS}

This work was in part supported by the Research and Development Applying Advanced Computational Science and Technology, Japan Science and Technology Corporation (JST) and the National Academy of Sciences of Ukraine.
[1] J.P. Hansen and I.R. McDonald, Theory of Simple Liquids, 2nd ed. (Academic Press, London, 1986).

[2] E. Lomba, G. Stell, J.A. Anta, and M. Alvarez, J. Chem. Phys. 97, 4349 (1992).

[3] C. Martin, E. Lomba, J.A. Anta, and M. Lombardero, J. Phys.: Condens. Matter 5, 379 (1993).

[4] S. Kambayashi and J. Chihara, Phys. Rev. E 50, 1317 (1994).

[5] J.J. Salacuse, A.R. Denton, and P.A. Egelstaff, Phys. Rev. E 53, 2382 (1996).

[6] H. Mori, K. Hoshino, and W. Watabe, J. Phys.: Condens. Matter 3, 9791 (1991).
[7] A. Baumketner and Y. Chushak, J. Non-Cryst. Solids 250, 354 (1999).

[8] J. Hafner, From Hamiltonians to Phase Diagrams (Springer, Berlin, 1987).

[9] S. Ichimaru and K. Utsumi, Phys. Rev. B 24, 7385 (1981).

[10] A. Busigin and C.R. Philips, Mol. Phys. 76, 89 (1992).

[11] Y. Rosenfeld and N.W. Ashcroft, Phys. Rev. A 20, 1208 (1979).

[12] Y. Waseda, The Structure of Non-crystalline Materials: Liquids and Amorphous Solids (McGraw-Hill, New York, 1980). 